\title{
Highlights on Dilepton and Photon Observables
}

\author{
Itzhak Tserruya ${ }^{\mathrm{a} *}$ \\ ${ }^{a}$ Weizmann Institute of Science, \\ Rehovot 76100, Israel
}

The highlights of Quark Matter 97 on dileptons and direct photons are presented.

\section{Introduction}

Evidence of enhanced dilepton production in $\mathrm{S}$ induced collisions at $200 \mathrm{~A} \mathrm{GeV}$ was first reported at the 95 Quark Matter Conference by the three experiments involved in the measurement of dileptons at the CERN SPS - CERES, HELIOS-3 and NA38 [1]. The enhancement is particularly large at low masses $\left(\mathrm{m}=0.2-1.0 \mathrm{GeV} / \mathrm{c}^{2}\right)$ but it is also significant at intermediate masses $\left(\mathrm{m}=1.5-2.5 \mathrm{GeV} / \mathrm{c}^{2}\right)$. In contrast, there was no clear evidence of a signal in the search of direct photons. Since then, these results have been at the focus of attention, triggering a strong interest mainly stimulated by the possibility that the low-mass excess could reflect the onset of chiral symmetry restoration [2]. Preliminary results with the $\mathrm{Pb}$ beam, presented at the 96 Quark Matter Conference by the CERES and NA50 experiments [3,4], show also an enhanced production of dileptons confirming at least qualitatively the results with the $\mathrm{S}$ beam. For recent reviews, see refs. [5,6]. In this Conference we have seen a wealth of new and interesting results, both on the experimental and theoretical fronts, related to the measurement of dileptons and photons at the CERN SPS. In this paper, I discuss the highlights in the field and I supplement what was presented in the talks with some comments and suggestions for future work.

\section{Low-mass Dileptons}

\subsection{Experimental Results}

Ravinovich presented a detailed analysis of low-mass electron pairs measured by CERES in $\mathrm{Pb}-\mathrm{Au}$ collisions at $160 \mathrm{~A} \mathrm{GeV}$ [7]. Fig. 11 shows the inclusive $e^{+} e^{-}$mass spectrum normalized to the charged-particle rapidity density. The spectrum is very similar to the one measured in S-Au collisions [8]. The yield is clearly enhanced compared to the predicted one on the basis of hadron decays shown by the thick solid line. The effect is most pronounced in the region from 300 to $700 \mathrm{MeV} / \mathrm{c}^{2}$ where the enhancement factor is $5.8 \pm 0.8$ (stat) \pm 1.5 (syst) but it also extends to higher masses. In the larger mass interval $0.2 \leq \mathrm{m} \leq 2.0 \mathrm{GeV} / \mathrm{c}^{2}$ the enhancement factor is $3.5 \pm 0.4$ (stat) \pm 0.9 (syst).

*e-mail: tserruya@ceres.weizmann.ac.il. Work supported by the Israeli Science Foundation, the MINERVA Foundation and the German-Israeli Foundation for Scientific Research and Development 


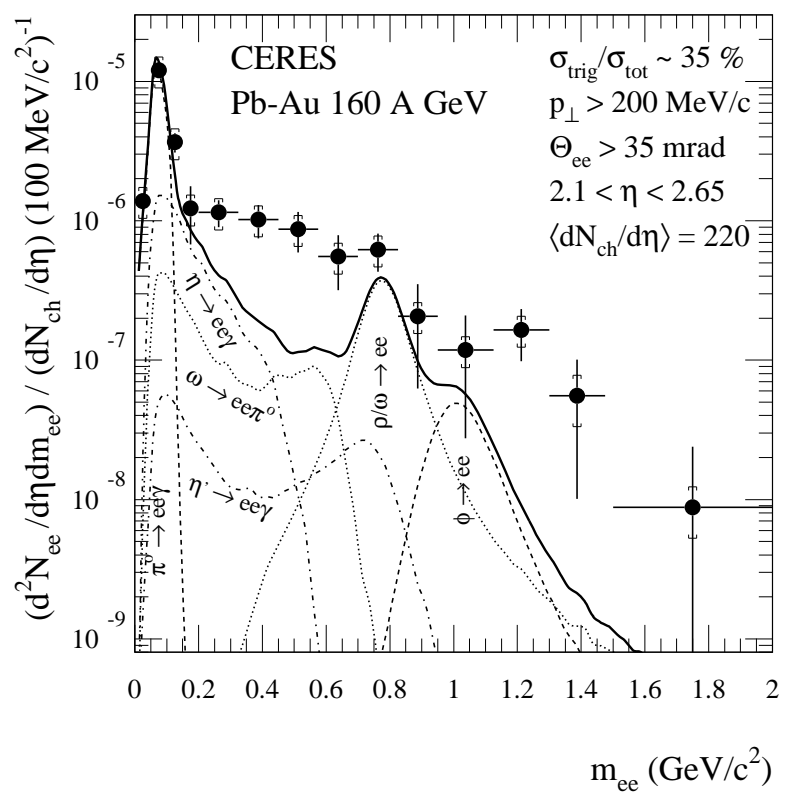

Figure 1. Inclusive $e^{+} e^{-}$mass spectrum measured by CERES in $160 \mathrm{~A} \mathrm{GeV} \mathrm{Pb-Au}$ collisions normalized to the observed charged-particle density. The Figure also shows the summed and individual contributions from hadronic sources [7].

Ravinovich presented also new results on multiplicity and $p_{t}$ dependence. In spite of the limited statistics, the results show [7] that the yield, integrated over mass, increases faster than linearly with multiplicity. Another significant feature is that the shape of the excess also changes with multiplicity as shown in Fig. 2. For masses $\mathrm{m}>200 \mathrm{MeV} / \mathrm{c}^{2}$ the yield is enhanced over the entire $p_{t}$ spectra, but it appears more pronounced at low $p_{t}$. This new information should be very valuable to further test and constrain the models put forward to explain the low-mass enhancement.

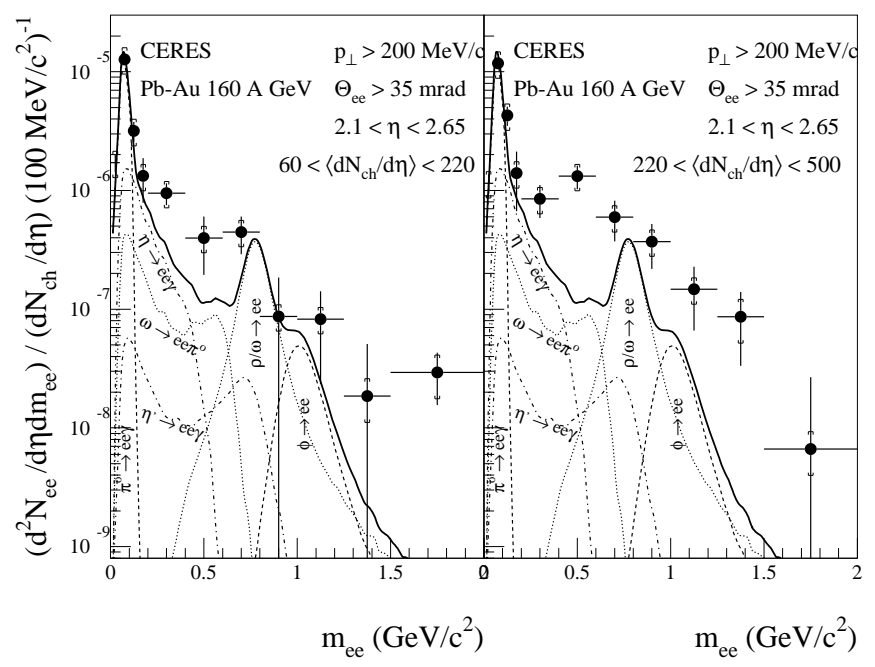

Figure 2. Inclusive $e^{+} e^{-}$mass spectra measured by CERES in $160 \mathrm{~A} \mathrm{GeV} \mathrm{Pb-Au} \mathrm{collisions}$ for low (left) and high (right) charged particle density. See also the caption of Fig. [1 [7]. 


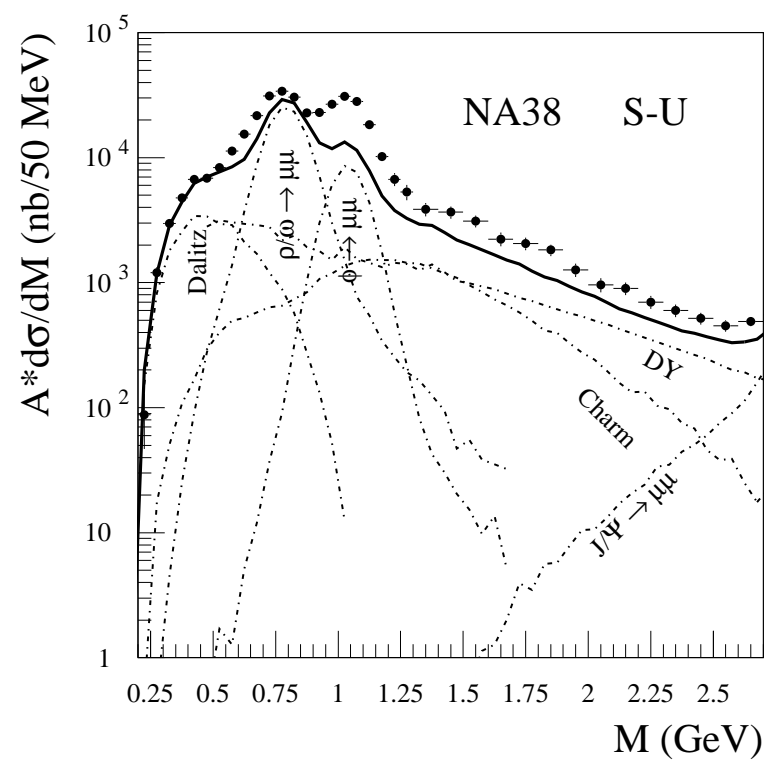

Figure 3. Inclusive $\mu^{+} \mu^{-}$mass spectra measured by NA38 in $200 \mathrm{~A}$ GeV S-U collisions. The thick line represents the summed yield of all known sources. The individual contributions are also shown [9].

Falco presented very interesting results of a systematic study focussed on the emission of low-mass dimuon pairs performed by NA38 and including p-U, S-S and S-U collisions at $200 \mathrm{~A} \mathrm{GeV} \mathrm{[9].} \mathrm{This} \mathrm{is} \mathrm{a} \mathrm{most} \mathrm{welcome} \mathrm{"new} \mathrm{arrival"} \mathrm{since} \mathrm{it} \mathrm{is} \mathrm{always} \mathrm{good} \mathrm{to} \mathrm{have} \mathrm{more}$ than one experiment looking at the same phenomena. Whereas the $\mathrm{p}-\mathrm{U}$ data are well reproduced by a cocktail of hadronic sources (with the somewhat uncertain extrapolation of the Drell-Yan contribution into low masses), the S data shows an enhancement of lowmass pairs. The enhancement is most apparent in the $\mathrm{S}-\mathrm{U}$ collision system and there it clearly extends over the intermediate mass region as illustrated in Fig.3.

There is a striking difference in the shape of the low-mass dilepton spectrum as measured by CERES and by NA38. A pronounced structure due to the resonance decays is clearly visible in the NA38 spectrum, whereas in the $\mathrm{S}$ and Pb CERES results the structure is completely washed out (see Figs.1, 2 and 4), raising the question of consistency between the two experiments. Resolution effects can be readily ruled out since the low-mass spectrum in p-Be and p-Au collisions measured by CERES with the same apparatus clearly shows the $\rho / \omega$ peak [10]. We note also that the two experiments cover nearly symmetric ranges around mid-rapidity $(\eta=2.1-2.65$ and $\eta=3-4$ in CERES and NA38 respectively). But there are also significant differences: CERES has a relatively low $p_{t}$ cut of $200 \mathrm{MeV} / \mathrm{c}$ on each track whereas NA38 is restricted to $m_{t}>0.9+2\left(y_{l a b}-3.55\right)^{2}$ $\mathrm{GeV} / \mathrm{c}^{2}$. Moreover, and probably more significantly, NA38 has no centrality selection in the trigger whereas the CERES data corresponds to the top $35 \%$ of the geometrical cross section. Given enough statistics it should be fairly easy for the two experiments to apply off-line common $m_{t}$ and centrality cuts thereby making possible a direct and meaningful comparison between their results. 


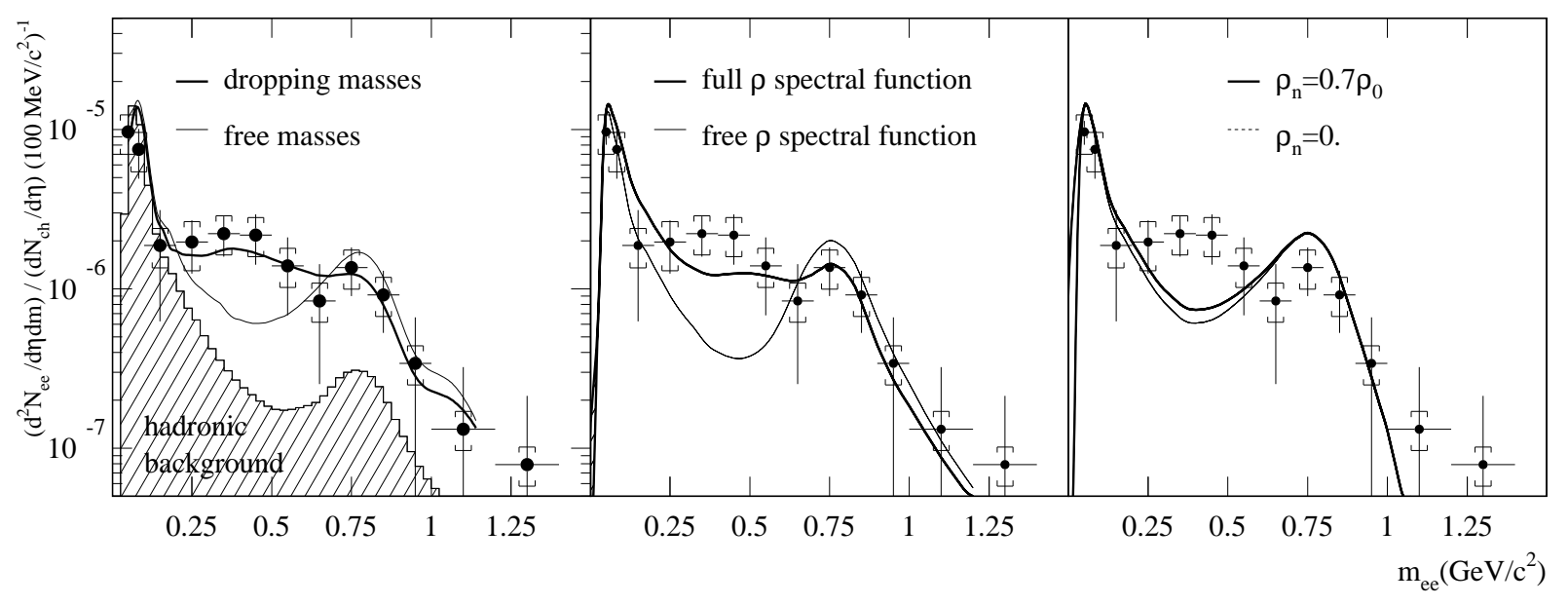

Figure 4. Inclusive $e^{+} e^{-}$mass spectra measured by CERES in $200 \mathrm{~A} \mathrm{GeV} \mathrm{S-Au} \mathrm{collisions}$ compared to calculations of Li, Ko and Brown [2] (left panel), Wambach [11,16] (middle panel) and Zahed [13,18] (right panel).

\subsection{Interpretations}

The S results of CERES and HELIOS-3 on low-mass dileptons have triggered a wealth of theoretical activity. A comprehensive theoretical review was given by Wambach [11] and very interesting results were presented by Li [12] and Zahed [13]. For an easier discussion and comparison between the different models I have plotted in Fig. 1 their results using the same scale and the same presentation of the S CERES data with statistical (vertical bars) and systematic (brackets) errors plotted independently. The histogram in the left panel represents the summed contribution from hadronic sources assuming a simple superposition of pp collisions. There is a consensus that an additional source beyond this simple assumption is needed. Furthermore, it is commonly recognized that the pion annihilation channel $\left(\pi^{+} \pi^{-} \rightarrow l^{+} l^{-}\right)$, obviously not present in pp collisions, has to be taken into account. This channel has been included in all recent calculations and although it accounts for a large fraction of the observed enhancement (the thin line in the three panels contain the pion annihilation in addition to the hadron decays) it is not sufficient and the calculations fail to reproduce the data in the mass region $0.2<m_{e^{+} e^{-}}<0.5$ $\mathrm{GeV} / \mathrm{c}^{2}$. These data have been quantitatively explained by taking into account in-medium modifications of the vector mesons. Li, Ko and Brown [2] were the first to propose and use a decrease of the $\rho$-meson mass in the hot and dense fireball as a precursor of chiral symmetry restoration. With this approach, an excellent agreement was achieved [2, 14] with the CERES data as demonstrated by the solid line in the left panel of Fig. $⿴$.

Wambach discussed in detail the results of a different approach, using a $\rho$-meson spectral function which takes into account the $\rho$ propagation in hot and dense matter, including in particular the pion modification in the nuclear medium and the scattering of $\rho$ mesons off baryons. This leads to a large broadening of the $\rho$-meson line shape and consequently to a considerable enhancement of low-mass dileptons. These calculations also reproduce

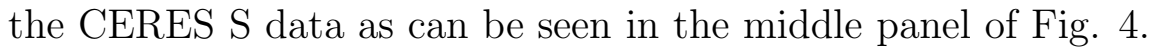

One should also notice that these two approaches reproduce equally well the S-W results of HELIOS-3 and the preliminary CERES results of $\mathrm{Pb}-\mathrm{Au}$ collisions 15 [17. 


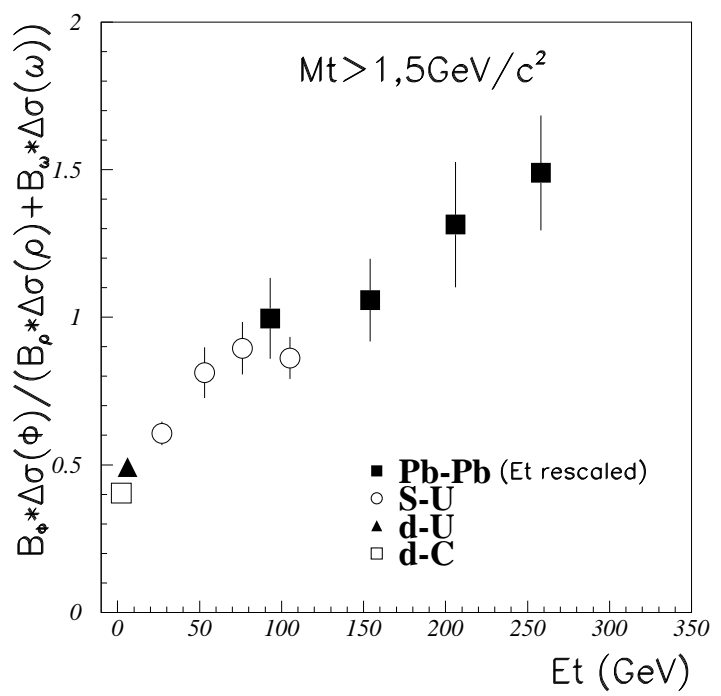

Figure 5. The $\phi /(\rho+\omega)$ ratio for various collision systems as a function of $\mathrm{E}_{t}$ measured by NA38/50 [19].

Their underlying physical picture is, however, very different. One case invokes chiral symmetry restoration which is an intrinsic property, whereas the second uses collision broadening which is a dynamic effect. More stringent tests are needed to help discriminate between the two approaches. The new results on multiplicity and $p_{t}$ dependences together with forthcoming results with reduced errors should be very helpful. Furthermore, the calculations themselves are not free of debate. In this context the results presented by Zahed at this Conference [13] are very interesting. His approach is in principle similar to the one of Wambach. He addresses the same physics of in-medium modifications of the $\rho$ spectral function, using on-shell chiral reduction formulas and enforcing known constraints, and yet, with a realistic nucleon density $\rho_{N}$, he fails to reproduce the lowmass data as shown in the right panel of Fig. 4 [13,18.

\section{Vector Mesons}

Jouan [19] presented new results from NA50 on the production of the vector mesons $\rho, \omega$ and $\phi$ in $\mathrm{Pb}-\mathrm{Pb}$ collisions. There is now an impressive set of data covering a variety of systems $\mathrm{d}-\mathrm{C}, \mathrm{d}-\mathrm{U}, \mathrm{S}-\mathrm{U}$ and $\mathrm{Pb}-\mathrm{Pb}$ collisions. The ratio $\phi /(\rho+\omega)$ for all these systems is shown in Fig. 5 as a function of $E_{t}$. The ratio increases approximately linearly with centrality and also with the size of the system, by almost a factor of four from d-C to $\mathrm{Pb}-$ $\mathrm{Pb}$ collisions. Historically, the data on vector meson production as measured by HELIOS and NA38 has always been presented in the form of the ratio $\phi /(\rho+\omega)$. The increase of the ratio was taken as a measure of the increase of the $\phi$-meson production -which is a very interesting feature in the context of strangeness enhancement- with the implicit assumption that the $\omega$ and $\rho$ meson production remains unaffected. However, since the $\rho$ meson is suspected to change its properties, as discussed in the previous section (either the width, or the mass or both), the amount of $\phi$ enhancement cannot be readily assessed. For that it would be more appropriate to see the yield of the $\phi$ and $(\rho+\omega)$ mesons plotted separately. 
6

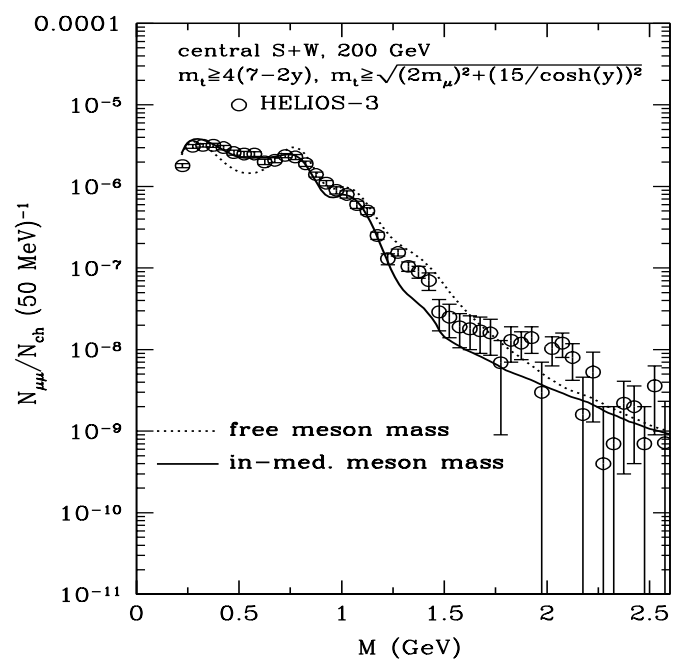

Figure 6. Calculations of Li with free and in-medium meson masees compared to the HELIOS-3 dimuon data [12].

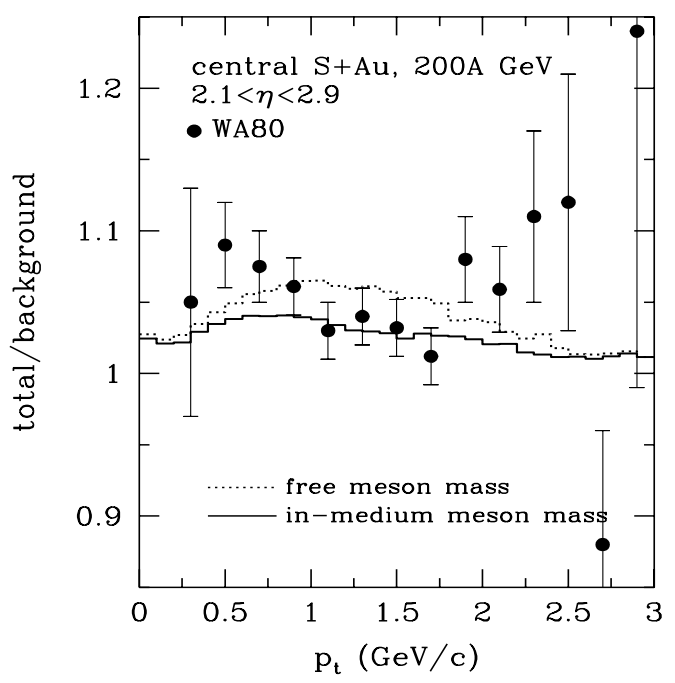

Figure 7. Comparison of the calculations of Li with the WA80 photon data in $\mathrm{S}-\mathrm{Au}$ collisions at $200 \mathrm{~A} \mathrm{GeV} \mathrm{[12].}$

\section{Intermediate-Mass Dileptons}

We have not seen new experimental results on dilepton production at intermediate masses $1.5<\mathrm{m}<3.0 \mathrm{GeV} / \mathrm{c}^{2}$ at this Conference. But we did see new calculations presented by Li [12] in comparison with the HELIOS-3 results on central S-W collisions at $200 \mathrm{~A} \mathrm{GeV}$. He considers on top of the physics background of Drell-Yan and open charm pairs, the thermal radiation of muon pairs resulting from secondary meson interactions including higher resonances and in particular the $\pi a_{1} \rightarrow l^{+} l^{-}$. His calculations are based on the same relativistic transport model used to calculate the low-mass dileptons discussed in the previous section. His results are presented in Fig. 6 showing the total yield (physics background + thermal yield) with the assumption of free masses (dotted line) and dropping vector meson masses (solid line). The latter leads to a much better agreement with the data at low masses (from 0.3 to $0.7 \mathrm{GeV} / \mathrm{c}^{2}$ ), as already mentioned in the previous section, whereas in the intermediate mass region the difference between free and in-medium meson masses with respect to the data is not so large. The calculations with free masses slightly overestimate the data whereas with dropping masses the situation is reversed. The intermediate mass region alone cannot justify the use of a dropping mass model, however it is important that this model can explain simultaneously the low and intermediate mass regions.

\section{Photons}

In contrast with the dilepton results, there is no clear evidence of enhancement in the measurements of real photons. All experiments performed to date with $\mathrm{O}$ and $\mathrm{S}$ beams have been able to establish only an upper limit for the production of thermal photons, which is of the order of $10-15 \%$ of the expected yield from hadron decays. The sensitivity is actually limited not by statistics but by the systematic errors. At this conference, WA98 has presented preliminary results on the search of direct photons in $\mathrm{Pb}-\mathrm{Pb}$ collisions which show a slightly larger effect, an excess of $\sim 20 \%$ over the hadronic background. 
Direct photons are expected to provide analogous information to thermal dileptons since real and virtual photons should carry the same physics information. Therefore, a simultaneous quantitative description of results on low-mass dileptons and direct photons within a single model is a significant step in establishing a consistent and reliable interpretation of experimental results. Li has shown a first attempt in this direction [20]. With the same fireball model including dropping masses used to explain the CERES and HELIOS-3 dilepton results he calculated the amount of direct photons in central S-Au collisions at $200 \mathrm{~A} \mathrm{GeV}$. The results are presented in Fig. 7 in the form of the ratio of the total photon yield to the hadronic background from $\pi^{0}$ and $\eta$ decays. The excess of direct photons is predicted to be a few percent of the hadronic background, in agreement with the experimental results and with a simple estimate based on order of magnitude considerations [1]. This sharpens the strict requirement imposed on the experiments to control the systematic errors down to the percent level in order to be able to observe direct photons.

\section{Acknowledgements}

It is a pleasure to thank all the speakers of the dilepton plenary and parallel sessions, and in particular A. Falco, D. Jouan, G.Q. Li, I. Ravinovich, J. Wambach and I. Zahed, for providing the material I needed for my oral and written presentations.

\section{REFERENCES}

1. I. Tserruya, Nucl. Phys. A590, 127c (1995).

2. G.Q. Li, C.M. Ko and G.E. Brown, Phys. Rev. Lett. 75, 4007 (1995), Nucl. Phys. A606, 568 (1996) and G.Q. Li, C.M. Ko, G.E. Brown and H. Sorge, Nucl. Phys. A611, 539 (1996).

3. G. Agakichiev et al., CERES Collaboration, Nucl. Phys. A610, 317c (1996).

4. E. Scomparin et al., NA50 Collaboration, Nucl. Phys. A610, 331c (1996).

5. A. Drees, Nucl. Phys. A610, 536 (1996).

6. I. Tserruya, Proc. Int. Summer School on the Physics of Quark Gluon Plasma, June 3-6, 1997, Hiroshima, Preprint nucl-ex/9801007.

7. I. Ravinovich, for the CERES Collaboration, these Proceedings.

8. G. Agakichiev et al., CERES Collaboration, Phys. Rev. Lett. 75, 1272 (1995).

9. A. Falco, for the NA38 Collaboration, these Proceedings.

10. G. Agakichiev et al., CERES Collaboration, Z. Phys. C (in press).

11. J. Wambach, these Proceedings.

12. G.Q. Li, these Proceedings.

13. I. Zahed, these Proceedings.

14. W. Cassing, W. Ehehalt and C.M. Ko, Phys. Lett. B363, 35 (1995) and W. Cassing, W. Ehehalt and I. Kralik, Phys. Lett. B377, 5 (1996).

15. C.M. Ko private communication.

16. R. Rapp, G. Chanfray and J. Wambach, Nucl. Phys. A617, 472 (1997).

17. W. Cassing, E.L. Bratkovskaya, R. Rapp and J. Wambach, Preprint nucl-th/9708020.

18. J.V. Steele, H. Yamagishi and I. Zahed, Preprint hep-ph/9704414.

19. D. Jouan, for the NA50 Collaboration, these Proceedings.

20. G.Q. Li and G.E. Brown, Preprint nucl-th/97060076. 